\title{
Determination of the TMD gluon density in a proton using recent LHC data
}

\author{
Nizami Abdulov ${ }^{1, *}$, Artem Lipatov ${ }^{2,3}$, Gennady Lykasov ${ }^{3}$, and Maxim Malyshev ${ }^{2}$ \\ ${ }^{1}$ Faculty of Physics, Lomonosov Moscow State University, 119991 Moscow, Russia \\ ${ }^{2}$ Skobeltsyn Institute of Nuclear Physics, Lomonosov Moscow State University, 119991 \\ Moscow, Russia \\ ${ }^{3}$ Joint Institute for Nuclear Research, 141980 Dubna, Moscow Region, Russia
}

\begin{abstract}
Unintegrated (or transverse momentum dependent, TMD) parton distributions in a proton are important in high-energy physics. Using the latest LHC data on the hadron production in $p p$ collisions, we determine the parameters of the initial TMD gluon density, derived in the framework of quark-gluon string model at the low scale $\mu_{0} \sim 1-2$ $\mathrm{GeV}$ and refine its large- $x$ behavior using data on the $t \bar{t}$ production at $\sqrt{s}=13 \mathrm{TeV}$. Then, by using the Catani-Ciafaloni-Fiorani-Marchesini (CCFM) evolution equation, we extend the obtained TMD gluon density to the whole kinematic region. We tested the proposed TMD gluon density to the inclusive Higgs production in different decay modes, tchannel single top production at the LHC and to the proton structure functions $F_{2}^{c}\left(x, Q^{2}\right)$ and $F_{2}^{b}\left(x, Q^{2}\right)$ in a wide region of $x$ and $Q^{2}$. Good agreement with the latest LHC and HERA data is achieved.
\end{abstract}

\section{Introduction}

Theoretical description of a number of processes at high energies and large momentum transfer containing multiple hard scales requires unintegrated, or transverse momentum dependent (TMD) parton density functions [1]. These functions encode nonperturbative information on a proton structure, including transverse momentum and polarization degrees of freedom. In the high-energy factorization [2], the production cross sections at low transverse momenta are governed by the nonperturbative input to the TMD parton density functions. The latter, being used as an initial condition for the subsequent QCD evolution, could play an important role in phenomenological applications [3-6]. At present, several approaches to determine the TMD gluon density in a proton are known in the literature. For example, in the Kimber-Martin-Ryskin (KMR) scheme developed at the leading order (LO) and next-to-leading order (NLO), the TMD quark and gluon densities are derived from the conventional parton distribution functions [7-9]. Also, the TMD quark and gluon densities in a proton were determined from fits to precision measurements of deep inelastic scattering cross sections at HERA and evolved by the Dokshitzer-GribovLipatov-Altarelli-Parisi (DGLAP) evolution with NLO splitting functions using the

\footnotetext{
*e-mail: nizami.abdulov@gmail.com
} 
parton branching method [10]. Alternatively, the parameters of the initial TMD gluon distribution were fitted from the precision HERA data on proton structure function $F_{2}\left(x, Q^{2}\right)$ in the range $x<5 \times 10^{-3}, Q^{2}>5 \mathrm{GeV}^{2}$, and $F_{2}^{c}\left(x, Q^{2}\right)$ at $Q^{2}>2.5 \mathrm{GeV}^{2}$ assuming the Gaussian-like dependence on the intrinsic gluon transverse momentum at $k_{T} \leq \mu_{0} \sim 2 \mathrm{GeV}$. The approach based on the Catani-Ciafaloni-Fiorani-Marchesini (CCFM) gluon evolution equation was used to determine the TMD gluon distribution in all kinematic region [11-14].

In [15-17] the initial TMD gluon density was derived in the framework of the soft quark-gluon string model by taking into account gluon saturation effects at low $Q^{2}$. The essential parameters were obtained from the best description of the inclusive spectra of hadrons produced in pp collisions at LHC energies in the midrapidity region at low transverse momenta $p_{T} \leq 4.5 \mathrm{GeV}$. Being used with the CCFM evolution, the predictions based on the proposed TMD gluon density describe well the HERA measurements of the proton structure functions $F_{2}^{c}\left(x, Q^{2}\right), F_{2}^{b}\left(x, Q^{2}\right)$ and $F_{L}\left(x, Q^{2}\right)$. Thereby, the connection between soft LHC processes and small- $x$ physics at HERA in a wide kinematical region was established. An important advantage of the approaches $[11,16,17]$ is that one can rather easily take into account a large piece of higher-order corrections, namely, part of NLO + NNLO $+\ldots$ terms containing leading $\log 1 / x$ enhancement of cross sections due to real initial state parton emissions absorbed into the CCFM evolution.

In this paper we continue previous studies and refine its large- $x$ behavior using the latest LHC data on the inclusive top quark pair production at $\sqrt{s}=13 \mathrm{TeV}$. Moreover, we test the parameters of the initial TMD gluon density using the recent NA61, LHC, and RHIC data for soft hadron production in $p p$ and $A A$ collisions obtained in a wide energy range. We extend the updated TMD gluon distribution to the whole range of the longitudinal momentum fraction $x$, transverse momentum $\mathbf{k}_{T}^{2}$, and hard scale $\mu^{2}$ numerically using the UPDFEVOLV package, which is the CCFM evolution code for TMD parton densities. The CCFM equation is the most suitable tool for our study since it smoothly interpolates between the small- $x$ Balitsky-FadinKuraev-Lipatov (BFKL) gluon dynamics and the conventional DGLAP one. Then, we supply the obtained TMD gluon density with the corresponding TMD valence and sea quark distributions calculated in the approximation, where the sea quarks occur in the last gluon splitting. Finally, we present several phenomenological applications of the proposed TMD parton densities to hard LHC processes sensitive to the quark and gluon content of the proton: proton structure functions $F_{2}^{c}$ and $F_{2}^{b}$, single top production and inclusive Higgs boson production at the LHC.

\section{The theoretical approach}

The determination of the parameters of the initial TMD gluon density in a proton can be split into the two almost independent pieces referring to the regions of small and large $x$. We consider first the small- $x$ region and start from the simple analytical expression for the starting TMD gluon distribution function at some fixed scale $\mu_{0} \sim$ $1-2 \mathrm{GeV}$. It can be presented in the form [17]

$$
f_{g}^{(0)}\left(x, \mathbf{k}_{T}^{2}, \mu_{0}^{2}\right)=\tilde{f}_{g}^{(0)}\left(x, \mathbf{k}_{T}^{2}, \mu_{0}^{2}\right)+\lambda\left(x, \mathbf{k}_{T}^{2}, \mu_{0}^{2}\right) f_{g}\left(x, \mathbf{k}_{T}^{2}\right)
$$

where $x$ and $\mathbf{k}_{T}$ are the proton longitudinal momentum fraction and two-dimensional gluon transverse momentum, respectively.

The first term $\tilde{f}_{g}^{(0)}\left(x, \mathbf{k}_{T}^{2}, \mu_{0}^{2}\right)$ was calculated within the soft QCD model: 


$$
\begin{aligned}
\tilde{f}_{g}^{(0)}\left(x, \mathbf{k}_{T}^{2}, \mu_{0}^{2}\right)=c_{0} & c_{1}(1-x)^{b}\left[R_{0}^{2}(x) \mathbf{k}_{T}^{2}+\right. \\
& \left.+c_{2}\left(R_{0}^{2}(x) \mathbf{k}_{T}^{2}\right)^{a / 2}\right] \exp \left(-\sqrt{R_{0}^{2}(x) \mathbf{k}_{T}^{2}}-d\left[R_{0}^{2}(x) \mathbf{k}_{T}^{2}\right]^{3 / 2}\right)
\end{aligned}
$$

where $R_{0}^{2}(x)=\left(x / x_{0}\right)^{\lambda} / \mu_{0}^{2}, c_{0}=3 \sigma_{0} / 4 \pi^{2} \alpha_{s}$.

The parameters $\sigma_{0}=29.12 \mathrm{mb}, \lambda=0.22, x_{0}=4.21 \times 10^{-5}, \alpha_{s}=0.2$ come from the Golec-Biernat-Wüsthoff (GBW) saturation model, while the other parameters $a$, $b, c_{1}, c_{2}$ and $d$ were fitted from LHC data on inclusive spectra of charged hadrons. The gluon density $\tilde{f}_{g}^{(0)}\left(x, \mathbf{k}_{T}^{2}, \mu_{0}^{2}\right)$ differs from the one obtained in the GBW model at $\left|\mathbf{k}_{T}\right|<1 \mathrm{GeV}$ and coincides with the GBW gluon at larger $\left|\mathbf{k}_{T}\right|>1.5 \mathrm{GeV}$.

The second term $f_{g}\left(x, \mathbf{k}_{T}^{2}\right)$ represents the analytical solution of the linear BFKL equation at low $x$ weighted with a matching function $\lambda\left(x, \mathbf{k}_{T}^{2}, \mu_{0}^{2}\right)$ :

$$
\begin{aligned}
& f_{g}\left(x, \mathbf{k}_{T}^{2}\right)=\alpha_{s}^{2} x^{-\Delta} t^{-1 / 2} \frac{1}{v} \exp \left[-\frac{\pi \ln ^{2} v}{t}\right], \\
& \lambda\left(x, \mathbf{k}_{T}^{2}, \mu_{0}^{2}\right)=c_{0}\left(\frac{x}{x_{0}}\right)^{0.81} \exp \left[-k_{0}^{2} \frac{R_{0}(x)}{\left|\mathbf{k}_{T}\right|}\right],
\end{aligned}
$$

where $t=14 \alpha_{s} N_{c} \zeta(3) \ln (1 / x), \Delta=4 \alpha_{s} N_{c} \ln 2 / \pi, v=\left|\mathbf{k}_{T}\right| / \Lambda_{Q C D}, k_{0}=1 \mathrm{GeV}$.

This term allows one to describe LHC measurements of inclusive charged hadrons up to $p_{T} \leq 4.5 \mathrm{GeV}$. It is important that the contribution from $f_{g}\left(x, \mathbf{k}_{T}^{2}\right)$ is only nonzero at $\left|\mathbf{k}_{T}\right| \ll \Lambda_{Q C D}(1 / x)^{\delta}$ with $\delta=\alpha_{s} N_{c}$, resulting in an average generated gluon transverse momentum of $\left\langle\left|\mathbf{k}_{T}\right|\right\rangle \sim 1.9 \mathrm{GeV}$. The latter value is close to the nonperturbative QCD regime that allows one to treat the TMD gluon density above as a starting one for the CCFM evolution.

From the description of the recent experimental data on inclusive $t \bar{t}$ production at $\sqrt{s}=13 \mathrm{TeV}$ (Fig. 1) and the CMS data on inclusive b-jet production we find that $b=10, d=0.4$ and overall normalization $N=0.27$ are more preferable to describe the distributions on the rapidity and transverse momentum of top quark pairs. These parameters also do not contradict our calculations and the experimental data on the pion transverse mass distribution in $\mathrm{Au}+\mathrm{Au}$ and $\mathrm{Pb}+\mathrm{Pb}$ collisions taken by the STAR Collaboration at the RHIC and the ALICE Collaboration at the LHC (see [18]). We extend the obtained TMD gluon density to the whole kinematic region using a numerical solution CCFM evolution equation. The obtained gluon density is labeled as Moscow-Dubna 2018, or MD'2018 and it is shown in Fig. 2 versus the longitudinal momentum fraction $x$ and transverse momentum $\mathbf{k}_{T}$ at different evolution scales. Additionally, we plot the TMD gluon distribution [11] (namely, the JH'2013 set 2), which is widely discussed in the literature and commonly used in applications. One can observe some difference in the absolute normalization and shape between both TMD gluon distributions. In particular, the $\mathbf{k}_{T}$ tail of the MD'2018 density function is the contribution due to the solution of the linear BFKL equation. Actually, it was needed to improve the description of the LHC data on charge hadron production in $p p$ collisions at $\sqrt{s}=7 \mathrm{TeV}$ and $2.5<p_{T}<4 \mathrm{GeV}$.

\section{Results}

Now we can apply the MD'2018 gluon density to several hard processes studied at hadron colliders. We consider the proton structure functions $F_{2}^{c}\left(x, Q^{2}\right)$ and $F_{2}^{b}\left(x, Q^{2}\right)$, 

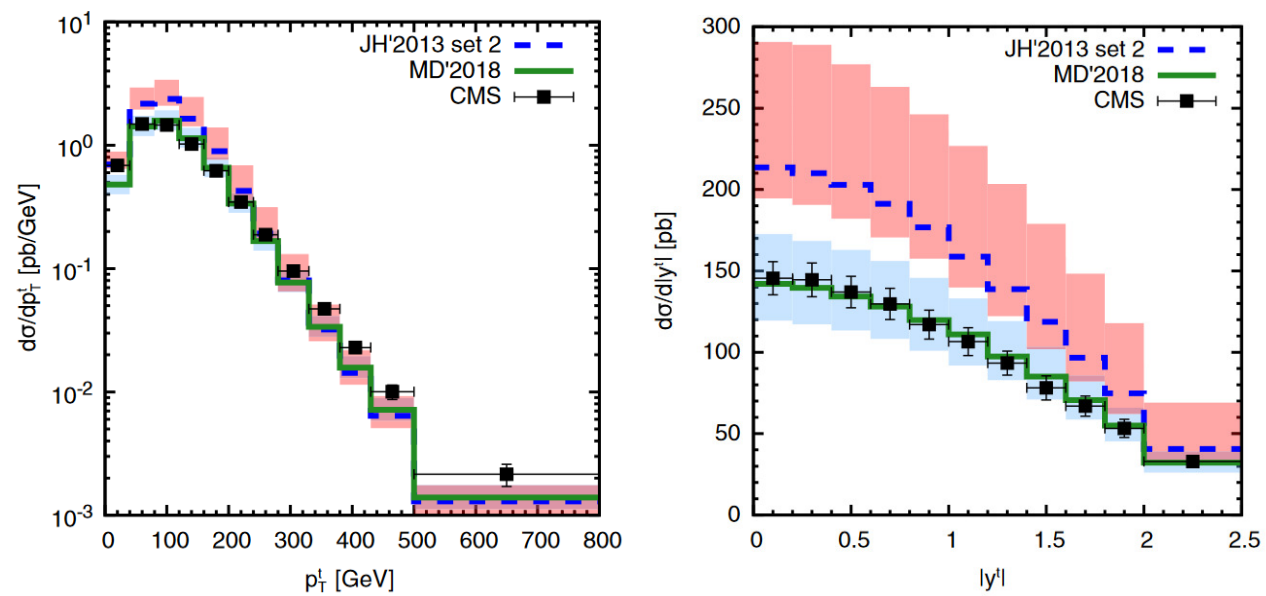

Figure 1. The transverse momentum and rapidity distributions of inclusive $t \bar{t}$ production in pp collisions at $\sqrt{s}=13 \mathrm{TeV}$. The green (solid) and blue (dashed) curves correspond to the predictions obtained using the MD'2018 and JH'2013 set 2 gluons, respectively. The shaded bands represent their scale uncertainties. The experimental data are from CMS [19]
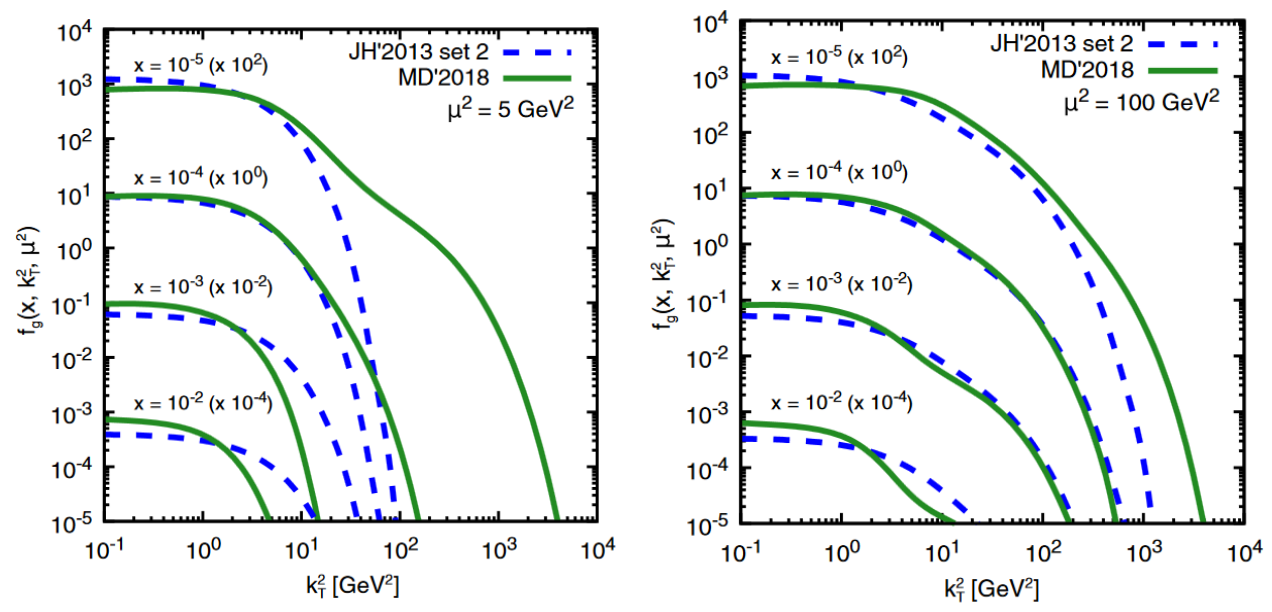

Figure 2. The TMD gluon densities in the proton calculated as function of the gluon transverse momentum $k_{T}^{2}$ at different longitudinal momentum fraction $x$ and $\mu^{2}$ values. The green (solid) and blue (dashed) curves correspond to the MD'2018 and JH'2013 set 2 gluon density functions, respectively

the t-channel single top production and inclusive Higgs production in different decay modes. All the details can be found in our previous work [18].

Results for the proton structure functions are shown in Figs. 3 and 4 . We find that the MD'2018 predictions for $F_{2}^{b}$ and $F_{2}^{c}$ are in reasonable agreement with the HERA data in a wide region of $x$ and $Q^{2}$, both in overall normalization and shape. It slightly overshoots the JH'2013 set 2 predictions at small $Q^{2}$ and low $x \leq 10^{-4}$. At larger $Q^{2}$ and moderate and/or large $x \geq 10^{-2}$, the JH'2013 set 2 gluon density function tends to overestimate the HERA data on the structure function $F_{2}^{b}$, which is due to the 
determination of input parameters of this gluon density at small- $x$ only. Therefore, the influence of the shape and other parameters of the initial nonperturbative gluon distribution on the description of the collider data is significant for a wide region of $x$ and $Q^{2}$. The MD'2018 gluon density, where all these parameters are verified by the description of LHC data, leads to a better agreement with the HERA data, which confirms the link between soft processes at the LHC and low- $x$ physics at HERA.

The results of our calculations for single $\bar{t}$ quark production in the $\mathrm{t}$ channel are presented in Fig. 5. We find that both the MD'2018 and JH'2013 set 2 gluon densities predict almost identical normalized cross sections, which agree with the CMS and ATLAS measurement quite well. However, the MD'2018 density results in a little smaller total cross section than the JH'2013 set 2 one that leads to somewhat better description of the data. Thus, the calculations endorse the usage of the MD'2018 gluon density for the evaluation of cross sections of processes with quite large- $x$ values involved. Note that the size of scale uncertainties of MD'2018 and JH'2013 set 2 calculations are rather close to each other in the kinematical region probed.

The results of our calculations of the cross sections of inclusive Higgs boson production obtained in different Higgs decay channels are shown in Figs. 6 and 7 in comparison with the latest LHC data. In the $H \rightarrow \gamma \gamma$ decay channel, we calculated the distributions on the diphoton pair transverse momentum $p_{T}^{\gamma \gamma}$ and absolute value of the rapidity $\left|y^{\gamma \gamma}\right|$. In the $H \rightarrow Z Z^{*} \rightarrow 4 l$ decay mode) at $\sqrt{s}=13$ decay channel, we calculated distributions on the Higgs transverse momentum $p_{T}^{H}$ and rapidity $\left|y^{H}\right|$. We find that both the MD'2018 and JH'2013 set 2 predictions reasonably agree with the data within the uncertainties for all considered kinematical observables, although the MD'2018 results lie a bit below the JH'2013 set 2 ones. Some tendency to underestimate the LHC data at large transverse momenta could be explained by the missing contributions from the weak boson fusion $\left(W^{+} W^{-} \rightarrow H\right.$ and $\left.Z Z \rightarrow H\right)$ and/or associated $H Z$ or $H W^{ \pm}$production [20], which become important at high $p_{T}$ and are not taken into account in our present consideration. The measured rapidity is well reproduced by our calculations. As one can see, despite the fact that both the MD'2018 and JH'2013 set 2 gluon distributions agree with the available data, the inclusive Higgs boson production at the LHC is very sensitive to the TMD gluon density in a proton, in particular, to the parameters of the initial TMD gluon distribution. It could be important to further constrain the latter.

\section{Conclusion}

We have refined a fit of the experimental data on the inclusive spectra of the charged particles produced in the central $p p$ and $A A$ collisions at the RHIC and the LHC to determine the TMD gluon density in a proton at the starting scale. The parameters of this fit do not depend on the initial energy in a wide energy interval. Using a numerical solution of the CCFM gluon evolution equation, we extended the derived TMD gluon density (denoted as Moscow-Dubna 2018 or MD'2018 set) to a whole kinematical region and supplied it with the relevant TMD valence and sea quark distributions. The latter was calculated in the approximation where the gluonto-quark splitting occurred at the last evolution step using the TMD gluon-to-quark splitting function. We achieved a good description of various HERA, RHIC and LHC data using the same set of parameters that confirms the link between soft processes at the LHC and low- $x$ physics at HERA. Finally, we demonstrated a significant influence of the initial nonperturbative gluon distribution on the description of the LHC data, 
which is important for further precise determination of the TMD quark and gluon densities in a proton.

Acknowledgements. We would like to thank S. P. Baranov and F. Hautmann for very useful discussions and important remarks. A. V. L. and M. A. M. are grateful to the DESY Directorate for the support in the framework of the Moscow- DESY project on Monte Carlo implementation for HERA-LHC. M. A. M. and N. A. A. were also supported by the Foundation for the Advancement of Theoretical Physics and Mathematics "Basis" (Grant Nos. 17-14-455-1 and 18-1-5-33-1). Part of this work was done by M. A. M. during his stay at DESY, funded by DAAD (program "Research Stays for University Academics and Scientists").

\section{References}

[1] R. Angeles-Martinez et al., Acta Phys. Pol. B 46, 2501 (2015)

[2] S. Catani, M. Ciafaloni, and F. Hautmann, Nucl. Phys. B 366, 135 (1991). J. C. Collins and R. K. Ellis, Nucl. Phys. B 360, 3 (1991). L. V. Gribov, E.M. Levin, and M. G. Ryskin, Phys. Rep. 100, 1 (1983). E.M. Levin, M. G. Ryskin, Yu.M. Shabelsky, and A. G. Shuvaev, Sov. J. Nucl. Phys. 53, 657 (1991)

[3] E. Avsar, Int. J. Mod. Phys. Conf. Ser. 04, 74 (2011). E. Avsar. arXiv:1203.1916

[4] S. M. Aybat and T. C. Rogers, Phys. Rev. D 83, 114042 (2011)

[5] B. I. Ermolaev, M. Greco, and S. I. Troyan, Eur. Phys. J. C 71, 1750 (2011).

B. I. Ermolaev, M. Greco, and S. I. Troyan, Eur. Phys. J. C 72, 1953 (2012)

[6] P. Kotko, K. Kutak, C. Marquet, E. Petreska, S. Sapeta, and A. Van Hameren, J. High Energy Phys. 09, 106 (2015)

[7] M. A. Kimber, A. D. Martin, and M. G. Ryskin, Phys. Rev. D 63, 114027 (2001)

[8] G. Watt, A. D. Martin, and M. G. Ryskin, Eur. Phys. J. C 31, 73 (2003)

[9] A. D. Martin, M. G. Ryskin, and G. Watt, Eur. Phys. J. C 66, 163 (2010)

[10] F. Hautmann, H. Jung, A. Lelek, V. Radescu, and R. Zlebcik, Phys. Lett. B 772, 446 (2017). F. Hautmann, H. Jung, A. Lelek, and V. Radescu, J. High Energy Phys. 01, 070 (2018)

[11] F. Hautmann and H. Jung, Nucl. Phys. B 883, 1 (2014)

[12] M. Ciafaloni, Nucl. Phys. B 296, 49 (1988)

[13] S. Catani, F. Fiorani, and G. Marchesini, Phys. Lett. B 234, 339 (1990).

S. Catani, F. Fiorani, and G. Marchesini, Nucl. Phys. B 336, 18 (1990)

[14] G. Marchesini, Nucl. Phys. B 445, 49 (1995)

[15] A. A. Grinyuk, A. V. Lipatov, G. I. Lykasov, and N. P. Zotov, Phys. Rev. D 93, 014035 (2016)

[16] A. V. Lipatov, G. I. Lykasov, and N. P. Zotov, Phys. Rev. D 89, 014001 (2014)

[17] A. A. Grinyuk, A. V. Lipatov, G. I. Lykasov, and N. P. Zotov, Phys. Rev. D 87, 074017 (2013)

[18] N. A. Abdulov, H. Jung, A. V. Lipatov, G. I. Lykasov, and M. A. Malyshev, Phys. Rev. D 98, 054010 (2018)

[19] CMS Collaboration, Phys. Rev. D 97, 112003 (2018)

[20] A. Szczurek, M. Luszczak, and R. Maciula, Phys. Rev. D 90, 094023 (2014)

[21] ZEUS Collaboration, J. High Energy Phys. 27, 127 (2014)

[22] H1 Collaboration, Eur. Phys. J. C 65, 89 (2010)

[23] H1 Collaboration, Eur. Phys. J. C 71, 1769 (2011). H1 Collaboration, Eur. Phys. J. C 72, $2252(2012)$ 
[24] ATLAS Collaboration, Eur. Phys. J. C 77, 531 (2017)

[25] CMS Collaboration, Report No. CMS-PAS-HIG-17-015, (2017)

[26] ATLAS Collaboration, arXiv:1802.04146

[27] CMS Collaboration, Report No. RCMS-PAS-HIG-16-041, (2016)

[28] ATLAS Collaboration, J. High Energy Phys. 10, 132 (2017). ATLAS Collaboration, Report No. ATLAS-CONF-2017-032, (2017) 


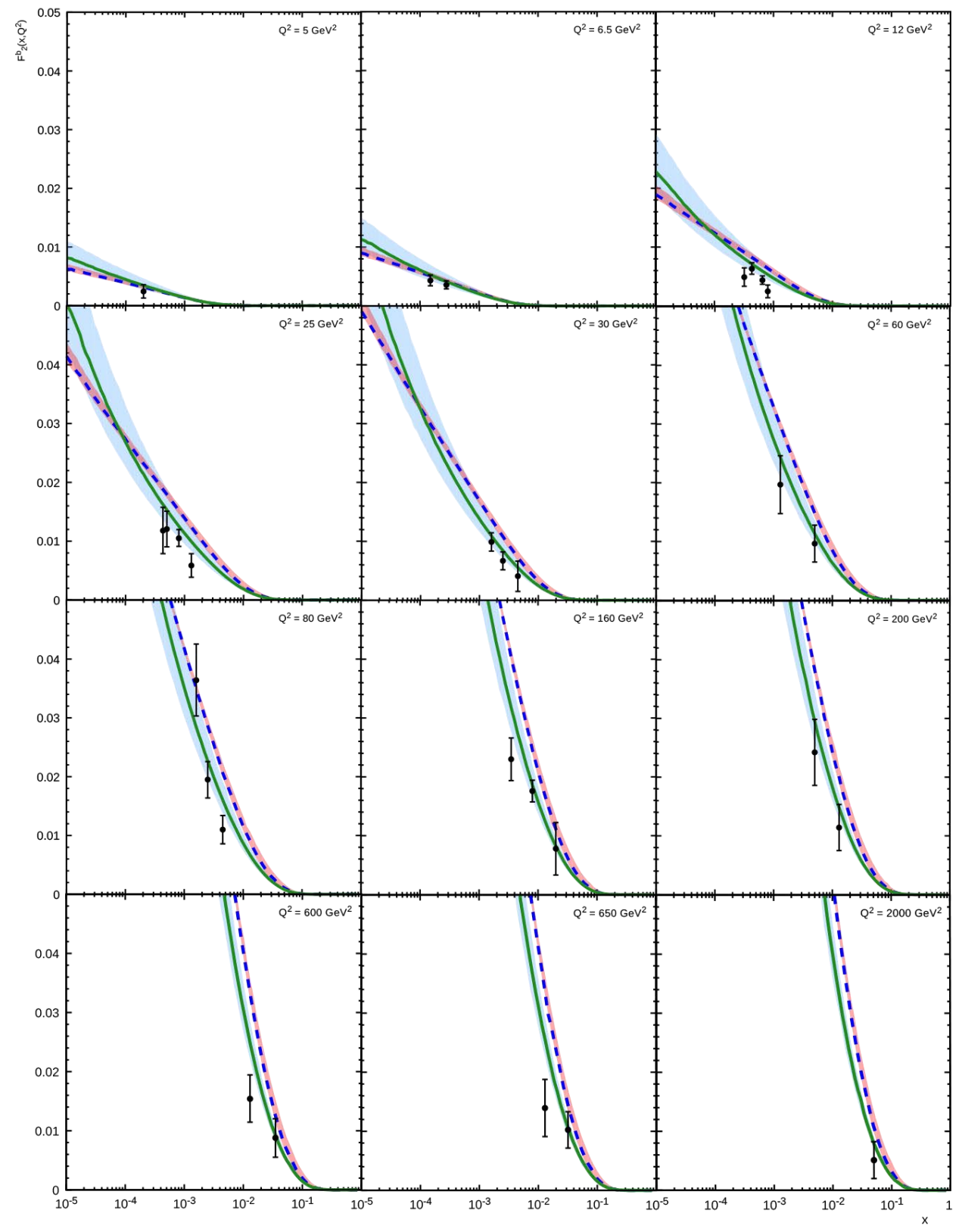

Figure 3. The beauty contribution to the proton structure function $F_{2}\left(x, Q^{2}\right)$ as a function of $x$ calculated at different $Q^{2}$. Notation of the histograms is the same as in Fig. 1 . The experimental data are from ZEUS [21] and H1 [22] 


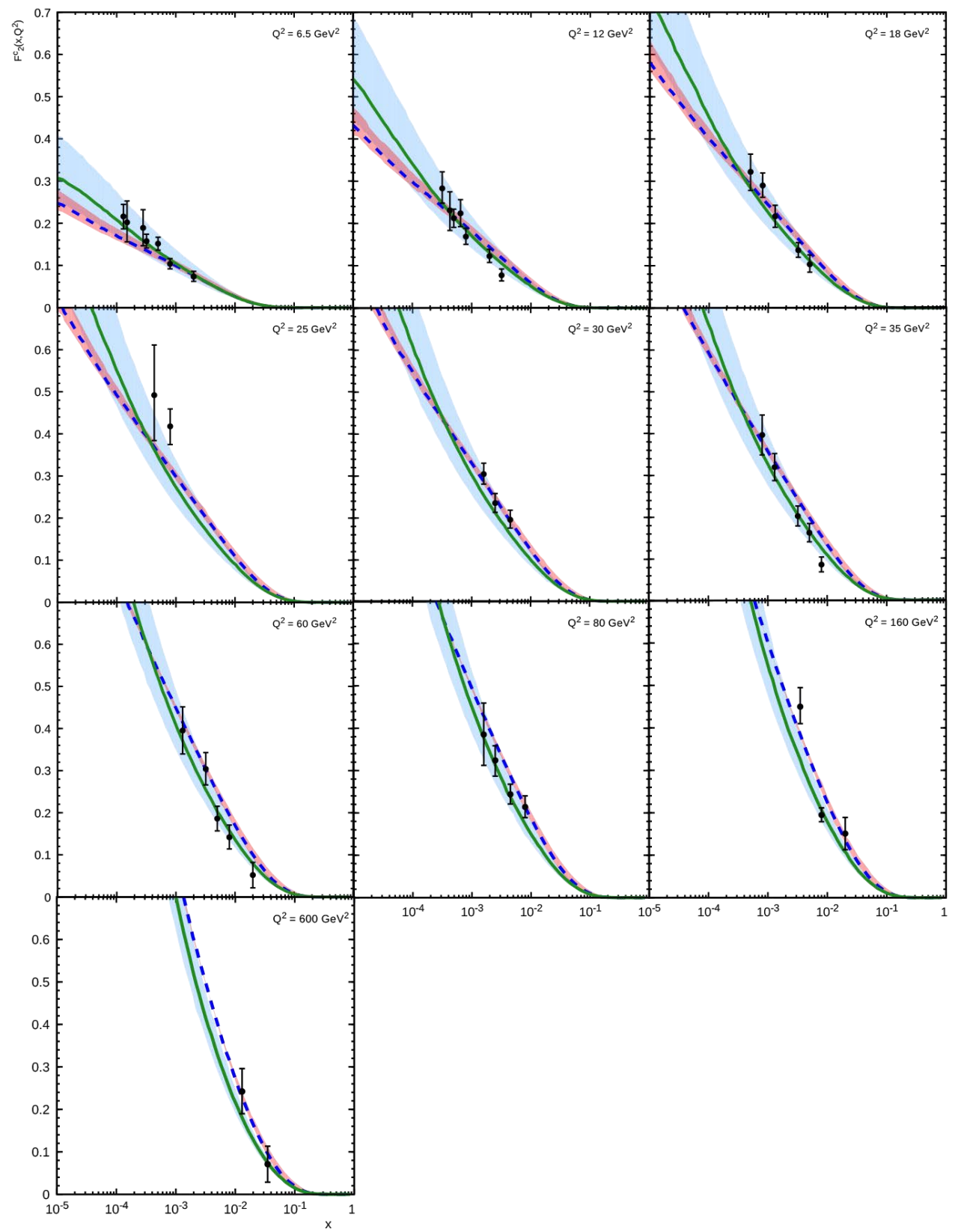

Figure 4. The charm contribution to the proton structure function $F_{2}\left(x, Q^{2}\right)$ as a function of $x$ calculated at different $Q^{2}$. Notation of the histograms is the same as in Fig. 1 . The experimental data are from ZEUS [21] and H1 [23] 

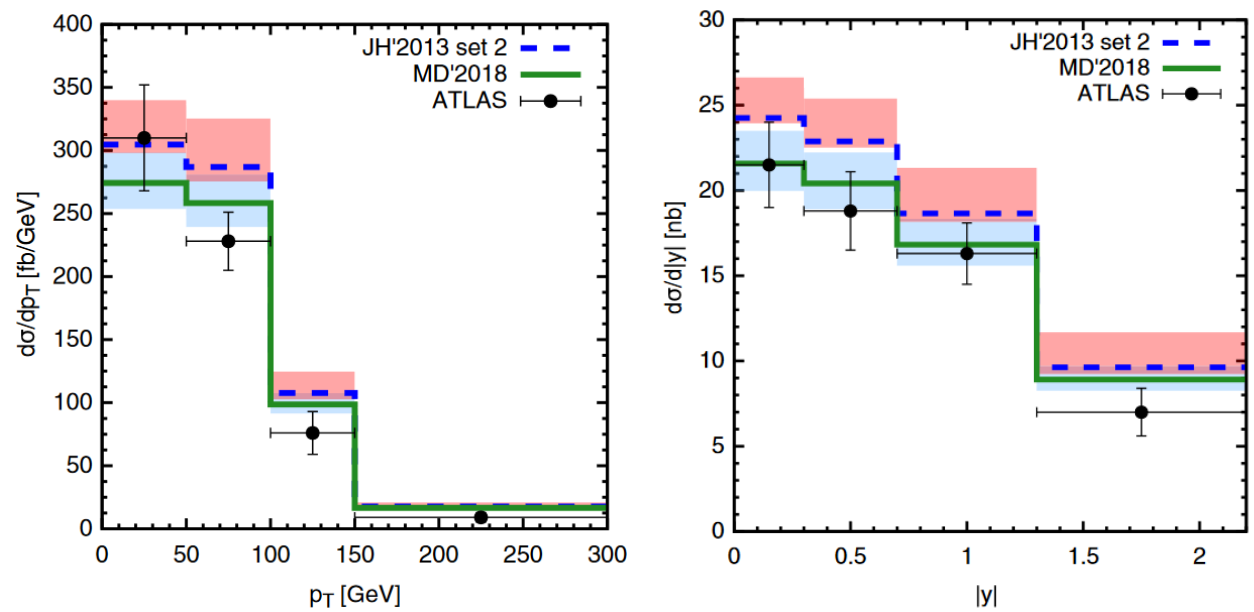

Figure 5. The differential cross sections of inclusive t-channel single antitop production at $\sqrt{s}=8 \mathrm{TeV}$ as functions of $\bar{t}$ quark transverse momentum and rapidity. Notation of the histograms is the same as in Fig. 1. The experimental data are from ATLAS [24]
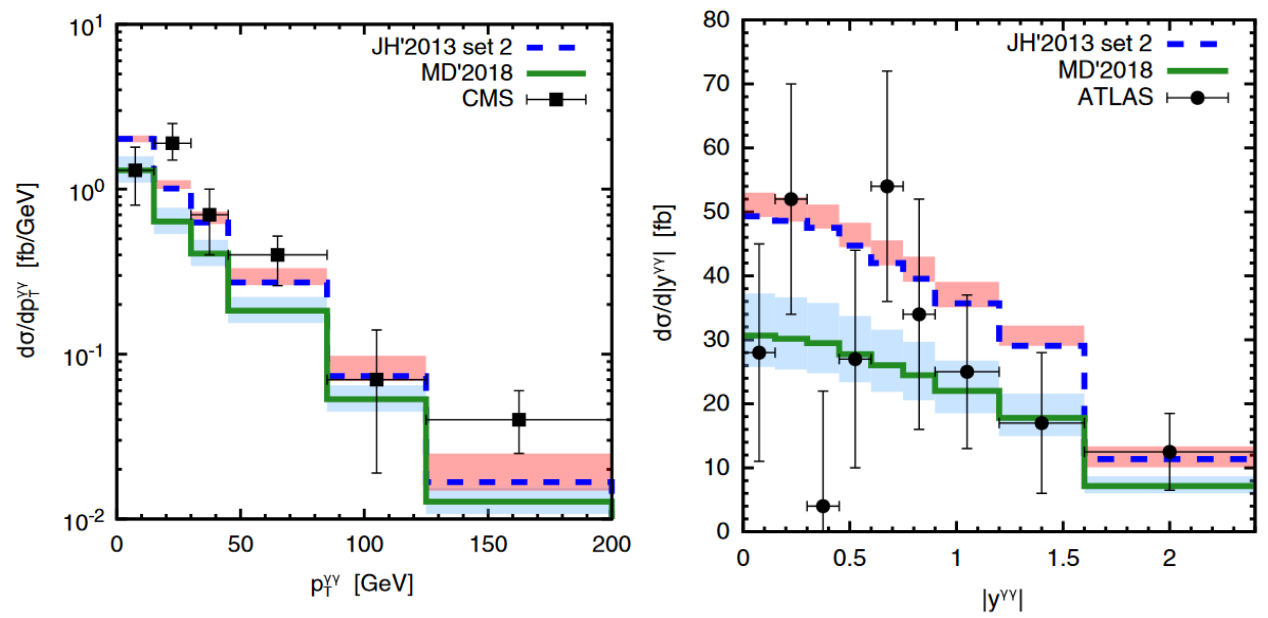

Figure 6. The differential cross sections of inclusive Higgs boson production (in the diphoton decay mode) at $\sqrt{s}=13 \mathrm{TeV}$ as functions of diphoton pair transverse momentum $p_{T}^{\gamma \gamma}$ and rapidity $\left|y^{\gamma \gamma}\right|$. Notation of the histograms is the same as in Fig. 1. The experimental data are from CMS [25] and ATLAS [26] 

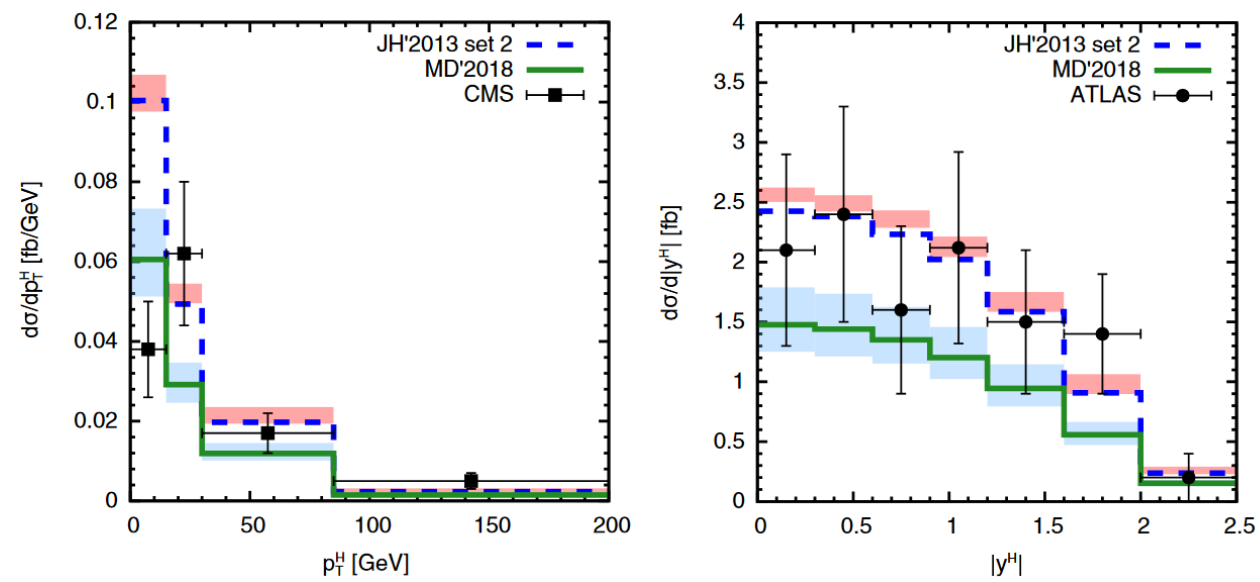

Figure 7. The differential cross sections of inclusive Higgs production (in the $H \rightarrow Z Z^{*} \rightarrow$ $4 l$ decay mode) at $\sqrt{s}=13 \mathrm{TeV}$ as functions of Higgs boson transverse momentum $p_{T}^{H}$ and rapidity $\left|y^{H}\right|$. Notation of the histograms is the same as in Fig. 1. The experimental data are from CMS [27] and ATLAS [28] 